\title{
Acesso ao serviço de emergência pelos usuários com crise hipertensiva em um hospital de Fortaleza, CE, Brasil
}

Access to the emergency service by clients with hipertensive crisis in a hospital in Fortaleza, CE, Brazil Aceso al servicio de urgencia por clientes con crisis de presión alta en un hospital de Fortaleza, CE, Brasil

\section{Ana Célia Caetano de Souza', Thereza Maria Magalhães Moreira', Maria Rocineide Ferreira da Silva', Paulo César de Almeida"l}

\author{
'Universidade Estadual do Ceará. Departamento de Enfermagem. Mestrado Acadêmico Cuidados Clínicos em Saúde \\ Grupo de Pesquisa Políticas, Saberes e Práticas em Saúde Coletiva. Fortaleza, CE \\ "Universidade Estadual do Ceará. Departamento de Estatística. Fortaleza, CE
}

Submissão: $21 / 08 / 2008$

Aprovação: 10/06/2009

\section{RESUMO}

A crise hipertensiva é uma elevação abrupta e sintomática da pressão arterial com risco de deterioração aguda de órgãos-alvo. Embora na literatura a prevalência do agravo seja de $1 \%$, os riscos de complicações e de morte associadas ao problema de saúde nos serviços de emergência têm revelado a necessidade de aprofundar Questões relacionadas ao acesso ao serviço de saúde. Trata-se de uma pesquisa de natureza Quantitativa, descritiva, realizada em um hospital público do município de Fortaleza, no período de abril a julho de 2006, com 118 pacientes. Os resultados demonstram Que a população com crise hipertensiva tem procurado os serviços de saúde, principalmente os de emergência, a fim de obter atendimento. O estudo indica o acesso como um elemento essencial para satisfação das necessidades dos usuários Que procuram os serviços de saúde, sendo o tempo de espera, a resolubilidade e o acolhimento parâmetros importantes na Qualidade do atendimento.

Descritores: Hipertensão; Serviços médicos de emergência; Enfermagem em emergência.

\section{ABSTRACT}

A hypertensive crisis is a sudden and symptomatic elevatation of blood pressure at risk of acute deterioration of target organs. Although the prevalence in the literature of the burden is $1 \%$, the risk of complications and death associated with the problem of health in the emergency services have revealed the need for deeper issues related to access to the service of health. It is a Quantitative and descriptive research, held in a public hospital Fortaleza city, between April and July of 2006, with 118 patients. The results show that people with hypertensive crisis has sought health services, especially the emergency, in order to get care. The study indicates access as a key element in meeting the needs of users who seek the services of health, and the waiting time, the resolution and the reception, important parameters on the euality of care.

Descriptors: Hypertension; Emergency medical services; Emergency nursing.

\section{RESUMEN}

La crisis hipertensiva es una elevación abrupta y sintomática de la presión arterial con riesgo de deterioro agudo de órganos-blanco. Aunque en las publicaciones la prevalencia del agravamiento sea de $1 \%$, los riesgos de complicaciones y de muerte asociadas al problema de salud en los servicios de emergencia han revelado la necesidad de profundizar en las cuestiones relacionadas al acceso al servicio de salud. Se trata de una investigación de naturaleza cuantitativa, descriptiva, realizada en un hospital público del municipio de Fortaleza, en el periodo de abril a julio de 2006, con 118 pacientes. Los resultados demuestran que la población con crisis hipertensiva ha buscado los servicios de salud, principalmente los de emergencia, para obtener atención. El estudio indica el acceso como un elemento esencial para la satisfacción de las necesidades de los usuarios que buscan los servicios de salud, siendo el tiempo de espera, la resolutividad y la acogida, los parámetros importantes en la calidad de la atención.

Descriptores: Hipertensión; Servicios médicos de urgencia; Enfermería de urgencia. 


\section{INTRODUÇÃO}

A crise hipertensiva é uma elevação abrupta e sintomática da pressão arterial com risco de deterioração aguda de órgãos-alvo (rim, cérebro, coração, retina e vasos sangüíneos), Que leva a pessoa aos centros de emergência, podendo envolver risco de morte eminente ou potencial ${ }^{(1,2)}$.

A enfermidade está dividida em urgência e emergência hipertensiva, e nos estudos brasileiros e estrangeiros, a prevalência gira em torno de $1 \%^{(1,3,4)}$. Embora essa prevalência do agravo seja pequena, os riscos de complicações e de morte associadas ao problema de saúde nos serviços de emergência têm revelado a necessidade de aprofundar Questões Que surgem acerca do acesso ao serviço de saúde.

O acesso ao serviço de saúde é um conceito complexo, onde se considera o grau de ajuste entre usuários e o sistema de saúde, observando-se a multidimensionalidade Que o compõe (disponibilidade dos serviços, acessibilidade, acolhimento e aceitabilidade) $)^{(5)}$.

A partir do Sistema Único de Saúde (SUS) se anuncia a necessidade de arranjos organizacionais em rede com potencialidade para garantir o acesso Qualificado dos usuários aos serviços de saúde ${ }^{(6)}$.

Nesse estudo, o acesso é focado como um dos elementos do sistema de saúde relacionado à organização dos serviços no Que se refere à assistência adequada ao usuário, no caso, iniciada na unidade de emergência com necessidade de continuidade do tratamento na clínica da atenção primária, uma vez Que a Quase totalidade da clientela é portadora de hipertensão arterial.

\section{METODOLOGIA}

Trata-se de um recorte de uma dissertação de mestrado ${ }^{(7)}$, Que se configurou como uma peseuisa descritiva-exploratória realizada em um hospital público do município de Fortaleza, no período de abril a julho de 2006. A população do estudo foi de 273 usuários e a amostra de 118 pessoas. Como critérios de inclusão no estudo adotaram-se: usuários com diagnóstico médico de crise hipertensiva ou Que apresentaram elevação abrupta e sintomática da pressão arterial; com níveis de pressão diastólica iguais ou superiores a $120 \mathrm{mmHg}$, com idade igual ou superior a 18 anos e orientados Quando da aplicação do instrumento de coleta de dados. Para análise dos dados foi utilizado o programa estatístico SPSS versão 13.0, onde foram calculados as medidas estatísticas média e desvio padrão e prevalência da crise hipertensiva no serviço. Foi utilizado um formulário contendo Questões relativas aos dados sóciodemográficos, clínico-epidemiológicos, de conhecimento da clientela e de acesso ao serviço de saúde. A pesquisa respeitou os preceitos éticos definidos na resolução 196/96 ${ }^{(8)}$ do Conselho Nacional de Saúde, sendo aprovada pelo Comitê de Ética em Pesquisa da Universidade Estadual do Ceará.

\section{RESULTADOS E DISCUSSÃO}

A amostra foi constituída por $53(44,9 \%)$ participantes do sexo masculino, $65(55,1 \%)$ do sexo feminino, com as faixas etárias predominantes de 42 a 53 anos e de 54 a 65 anos com $37,3 \%$ e
$29,7 \%$, respectivamente, e baixo nível de escolaridade analfabeto/ fundamental incompleto, representados por $78,8 \%$ da clientela. $\mathrm{O}$ tempo de permanência das pessoas no serviço de emergência da instituição variou de 75 a 1430 minutos, com média de 319,22 $\pm 270,34$. Esse tempo foi contabilizado desde a realização da ficha de atendimento até a alta dada pelo médico, e dependeu de alguns fatores diretamente implicados no atendimento. A necessidade de realização de exames complementares, como sangue, tomografia de crânio, eletrocardiograma; a redução lenta da pressão arterial; a melhora gradual dos sintomas, bem como a grande Quantidade de pessoas nos serviços de emergência com problemas não urgentes prolongaram esse tempo.

Corroborando os dados encontrados, estudo realizado em departamento de acidentes e emergência aponta que o maior tempo de permanência nesses serviços está relacionado à necessidade de realização de raioX, de encaminhamentos para especialistas e de maior permanência das pessoas na observação devido ao tratamento realizado ${ }^{(9)}$.

Pode-se afirmar Que uma média de 300 minutos no tempo de permanência é relativamente grande para o atendimento, mesmo com a realização de exames, e Que esse tempo poderia ser reduzido se houvesse uma priorização no atendimento à clientela com crise hipertensiva.

É importante considerar que os casos de emergência hipertensiva implicam em um tempo de permanência maior em virtude da necessidade de monitoramento dos sinais vitais, nível de consciência, perfusão renal, dentre outros. A espera por uma vaga na Unidade de Terapia Intensiva (UTI) também prolonga o tempo de permanência no serviço de emergência. Dados do hospital revelam Que no período de janeiro de 2002 a julho de 2006, a média do tempo de permanência das pessoas com diagnóstico de emergência hipertensiva e aguardando leito de UTI foi de 1 ,42 dias e o número de óbitos foi em torno de 14\%.

O horário no Qual as pessoas foram atendidas na emergência com crise hipertensiva no hospital em estudo não teve variação significativa, sendo praticamente eqüitativo nos três turnos de atendimento, e terças, Quartas e sextas-feiras foram os dias da semana onde aconteceu maior número de casos das crises hipertensivas.

A tabela I aborda o acesso ao serviço de saúde pelos sujeitos da pesquisa. A maioria $(77,1 \%)$ utilizava as diversas unidades disponíveis no sistema de saúde a fim de obter o atendimento. Entre os Que buscaram outros serviços de saúde além do hospital, Quase metade $(48,8 \%)$ recorreu a unidade básica de saúde; cerca de um terço (3 I\%) buscou outros hospitais da rede pública; $4,8 \%$ (Quatro) receberam atendimento nos centros de saúde; 2,4\% (dois) utilizaram clínicas particulares; 10,7\% (nove) procuraram atendimento nas unidades básicas de saúde, bem como em outros hospitais, e 2,4\% (dois) mencionaram o atendimento em outros hospitais, farmácias e outras unidades de saúde.

Percebeu-se Que a maior procura dos usuários pelos serviços se dá nas unidades básicas de saúde, o Que pode estar relacionado a seu acometimento por hipertensão, preconizando o acompanhamento na atenção básica, atualmente municipalizado. É válido ressaltar Que a recente inserção do Programa Saúde da Família (PSF) na atenção primária na área de abrangência do hospital tem promovido maior procura pelas unidades básicas de saúde. 
Tabela l. Distribuição do número de pacientes segundo acesso ao serviço de saúde. Fortaleza, 2006.

\begin{tabular}{|c|c|c|c|}
\hline CARACTERÍSTICAS & $\mathbf{N}$ & $\%$ & Média \pm DP \\
\hline \multicolumn{4}{|l|}{ Utiliza outro tipo de serviço de saúde $(n=118)$} \\
\hline Sim & 91 & 77,1 & \\
\hline Não & 27 & 22,9 & \\
\hline \multicolumn{4}{|l|}{ Qual $(n=84)$} \\
\hline Posto de saúde & 41 & 48,8 & \\
\hline Outro hospital & 26 & 31,0 & \\
\hline Centro de Saúde & 4 & 4,8 & \\
\hline Clínica Particular & 2 & 2,4 & \\
\hline Posto de Saúde + Outro hospital & 9 & 10,7 & \\
\hline Outro hospital + Farmácia + centro de saúde & 2 & 2,4 & \\
\hline \multicolumn{4}{|l|}{ Em Que ocasiões $(n=93)$} \\
\hline Periodicamente para prevenir doenças e p/tratamento & 10 & 10,8 & \\
\hline médico & 21 & 22,6 & \\
\hline Somente em caso de urgência & 17 & 18,2 & \\
\hline $\mathrm{P} /$ Receber medicação & 3 & 3,2 & \\
\hline p/Nerificar a pressão arterial & 45 & 48,9 & \\
\hline Mais de uma opção & & & \\
\hline \multicolumn{4}{|l|}{ Tempo (em minutos) de espera para receber atendimento nesse } \\
\hline serviço $(n=118)^{*}$ & 46 & 39,0 & Média: 48,18 \\
\hline $00-10$ & 35 & 29,7 & DP: 55,77 \\
\hline $11-30$ & 20 & 16,9 & \\
\hline $31-90$ & 17 & 14,4 & \\
\hline $91-120$ & & & \\
\hline \multicolumn{4}{|l|}{ Tempo (em minutos) de espera para receber atendimento em outro } \\
\hline $00-09$ & 17 & 25 & Média: 66,67 \\
\hline $10-20$ & 23 & 33,8 & DP: 213,57 \\
\hline $21-60$ & 12 & 12,9 & \\
\hline $61-?$ & 50 & 42,4 & \\
\hline ignorado & & & \\
\hline \multicolumn{4}{|l|}{ Alguma vez procurou o serviço de saúde e não foi atendido ( $\mathrm{n}=$} \\
\hline 118) & 23 & 19,5 & \\
\hline Sim & 95 & 80,5 & \\
\hline Não & & & \\
\hline \multicolumn{4}{|l|}{ Quantas vezes $(n=23)$} \\
\hline I vez & 6 & 26 & \\
\hline 2 a 10 vezes & 7 & 30,4 & \\
\hline \multicolumn{4}{|l|}{ Foi bem atendido $(n=118)$} \\
\hline Sim & 108 & 91,5 & \\
\hline Não & 6 & 5,1 & \\
\hline Às vezes & 4 & 3,4 & \\
\hline
\end{tabular}

Total de participantes $N=/ / 8$.

Onde: $p<0,05$

Quase um terço (31\%) dos participantes buscou para seu atendimento somente o hospital onde foi realizado o estudo. Esse fato pode ser explicado pela existência de fatores ligados ao acesso ao serviço de saúde tais como: a confiança Que depositam na instituição e/ou nos profissionais Que realizam o atendimento; a proximidade do hospital a suas residências, facilitando o deslocamento; mais recursos materiais disponíveis e maior resolutividade, pois a unidade possui além do serviço de emergência, laboratório 24 horas, farmácia e serviços de imageamento (radiologia, ultra-sonografia, endoscopia, ecocardiograma e mamografia), dentre outros.
Ramos e Lima ${ }^{(10)}$ referem Que o acesso ao serviço de saúde diz respeito à distância entre o domicílio do indivíduo e a unidade; o tempo gasto para o deslocamento e os meios utilizados com essa finalidade; dificuldades para conseguir o atendimento traduzido pela presença de filas, tempo e local de espera; o tratamento que o usuário recebe dos diversos profissionais e a priorização no atendimento de casos urgentes, bem como a possibilidade de agendamento prévio.

A ocorrência de crise hipertensiva tem possibilitado a procura pelas instituições hospitalares, uma vez Que é um problema de saúde Que pode acometer o indivíduo em qualquer horário. Além disso, a 
presença de sintomatologia e a possibilidade de gravidade estimulam a procura pelo serviço de emergência. A crença por parte da clientela de Que a emergência é um serviço melhor equipado e com recursos humanos "mais capacitados" para o atendimento também pode contribuir na busca pelo serviço. Tal crença tem fundamento nas superespecialidades da saúde acontecida a partir da década de $70^{\text {(11). }}$. A utilização inapropriada dos hospitais pela população também pode estar relacionada a problemas de acesso, como as falhas na organização dos serviços de atenção básica.

Uma pesquisa social realizada nos anos de 1989 e 1996-1997 sobre a utilização de serviços de saúde no Brasil indica um aumento na procura de hospitais pela população brasileira, principalmente no Nordeste, em decorrência do aumento na utilização de pronto-socorros ou serviço ambulatorial hospitalar ${ }^{(5)}$.

Percebeu-se que 10,7\% (nove) dos participantes buscaram atendimento em outros hospitais e unidades básicas de saúde. Na tentativa de resolver seus problemas de saúde, as pessoas passam a procurar atendimento nas várias unidades de saúde existentes no sistema. O consumo do serviço de saúde particular foi de $2,4 \%$ (dois) do total, sendo uma utilização pequena, explicada pela baixa renda familiar das pessoas envolvidas na pesquisa, Que dificilmente poderiam comprar serviço de saúde.

Entre os Que utilizaram outras unidades de saúde, 18,2\% (21) das pessoas se deslocavam para outro serviço, principalmente para a unidade básica de saúde com a finalidade de adeuirir medicamentos, particularmente os anti-hipertensivos, ocorrência explicada pelo fato de $95,4 \%$ dos usuários serem portadores de hipertensão arterial. Cerca de 10,8\% (10) relataram Que buscam os serviços de saúde periodicamente para prevenir doenças e realizar tratamento médico; Quase um terço $(22,6 \%)$ utilizava outro serviço somente em caso de urgência; 3,2\% (três) usavam para verificar a pressão arterial e Quase metade $(48,9 \%)$ acessava os serviços por mais de um motivo.

Percebeu-se que é preocupante o número de usuários $(22,6 \%)$ Que procura o serviço de saúde somente em caso de urgência, demonstrando falhas no acesso ao serviço de saúde, possibilitando o desencadeamento de sucessivas crises hipertensivas.

Os motivos pelos Quais as pessoas acessaram outras unidades de saúde do sistema foram diversos, sendo que Quase a metade $(48,9 \%)$ dos usuários procurava outros serviços por mais de um motivo. A necessidade de obter os medicamentos disponibilizados atualmente nas unidades de atenção básica em Fortaleza foi uma das principais causas desse deslocamento. Em um estudo sobre acesso e acolhimento em unidade de saúde, o autor coloca Que os usuários realizam uma verdadeira migração entre várias unidades de saúde em busca de medicamentos, muitas vezes indisponíveis no local onde ocorreu o atendimento ${ }^{(10)}$.

Outro fator Que leva as pessoas a utilizar outras instituições para o atendimento está relacionado à prevenção de doenças e à necessidade de tratamento médico. $\mathrm{O}$ tratamento dos problemas de saúde na clientela está ligado à inserção no programa de hipertensão e diabetes.

É importante salientar Que durante o período de realização da coleta de dados, dentre as unidades básicas de saúde próximas à área de abrangência do hospital Que podiam realizar o atendimento dessa clientela, apenas uma possuía equipe composta por médico, enfermeiro e auxiliar de enfermagem. Portanto, as unidades básicas não ofereciam o atendimento preconizado pelo Ministério da Saúde, Que se constitui de uma equipe composta por médico, enfermeiro, auxiliar de enfermagem, e Quatro a seis agentes de saúde ${ }^{(12)}$.

O tempo de espera do usuário para receber o atendimento ao chegar ao hospital do estudo com crise hipertensiva variou de 0- 120 minutos, com média de 48,18 $\pm 55,77$, sendo significativa a relação tempo de espera no nosocômio onde foi realizada a pesquisa e o fato de ter sido bem atendido. Cerca de 39\% (46) da clientela relatou ter esperado até 10 minutos para ser atendida; Quase um terço 29,7\% (35) aguardou de 11-30 minutos para o atendimento; 16,9\% (20) disseram Que o serviço levou de 31-90 minutos, e 14,4\% (17) mencionaram Que o tempo de espera variou de 91 - 120 minutos. Sabese Que o tempo de espera é um dos parâmetros importantes do acesso ao serviço de saúde e indica Qualidade no atendimento.

Percebeu-se que o tempo de espera relatado por mais de um terço dos participantes (39\%) foi de até dez minutos, demonstrando um atendimento relativamente rápido.

A avaliação do indivíduo com diagnóstico médico de crise hipertensiva deve ser feita de maneira rápida, no sentido de diferenciar as urgências das emergências hipertensivas para seu prontoatendimento, evitando o agravamento do Quadro clínico. A necessidade de instituir o tratamento médico correto, nas primeiras vinte Quatro horas, na urgência hipertensiva e, em menos de uma hora, na emergência se reveste de fundamental importância no tratamento da crise hipertensiva, bem como na prevenção ou limitação de lesões em órgãosalvo ${ }^{(13)}$. Portanto, um tempo de espera superior a sessenta minutos pode ser extremamente maléfico para a pessoa com crise hipertensiva, podendo causar inclusive a morte.

O tempo de espera para receber atendimento em outro serviço de saúde com crise hipertensiva variou de 0-61 minutos ou mais, com média de $66,67 \pm 213,57$. Menos de um Quarto (23,5\%) relatou ter esperado até nove minutos pelo atendimento; um Quarto (25\%) aguardou de dez a vinte minutos; um terço $(33,8 \%)$ esperou de vinte e um a sessenta minutos e 12,9\%(12) disseram Que o serviço levou sessenta e um minutos ou mais para realizar esse atendimento. Portanto, o tempo de espera do usuário com diagnóstico de crise hipertensiva em outro serviço de saúde foi maior do Que no hospital do estudo, com predominância na variação de 21-60 minutos.

Estudo realizado em departamento de emergência de um hospital de atenção secundária no México encontrou Que o tempo de espera para receber atendimento é determinante para maior satisfação do usuário Que utiliza esse serviço e Que o tempo de espera diminuiu Quando foi implantado o serviço de triagem de acordo com o grau de emergência envolvido. Outro achado importante é que a satisfação do usuário depende da relação entre o profissional de saúde e o usuário do serviço, e do tempo de espera durante a visita ao serviço ${ }^{(14)}$.

Esse estudo encontrou, ainda, Que o atendimento baseado na classificação de risco considera Que a categoria 1 (clientes com risco de morte eminente) deve receber atendimento de forma imediata; clientes na categoria 2 (pessoas com problemas muito urgentes e que estejam seriamente lesionadas) necessitam ser atendidos em menos de 10 minutos e na categoria 3 (indivíduos com problemas urgentes), o atendimento não deve exceder a 30 minutos $^{(14)}$

Pode-se observar Que 31,1\% da nossa clientela esperou para 
ser atendida no hospital do estudo por mais de 30 minutos, embora estejam inseridos nas categorias 1, 2 e 3 segundo a classificação de risco em clientes com emergência e urgência. Paine ${ }^{(9)}$ afirma Que o tempo de espera contabilizado em departamento de acidentes e de emergência foi de 60 minutos, sendo Que a avaliação realizada pela enfermeira leva até 10 minutos e o atendimento feito pelo médico até 60 minutos.

A tabela demonstra Que Quase a totalidade $(91,5 \%)$ relatou ter sido bem atendida pelo serviço de saúde. Entre os Que relataram não ter sido bem atendidos, $5,1 \%$ (seis) não receberam atendimento pelo menos uma vez e $5,9 \%$ (sete) deixaram de ser atendidos entre duas a dez vezes.

A falta de atendimento é um dos parâmetros Que avalia de forma negativa os serviços de saúde. $\mathrm{O}$ fato de ter o atendimento negado promove no indivíduo desconfiança e descrédito na unidade de saúde.

A compreensão do bom atendimento é um processo subjetivo e depende da avaliação individual. No entanto, ser bem atendido está relacionado a Questões de satisfação, como ser bem recebido pelos profissionais de saúde, tempo de aguardo menor que o esperado, receber informações precisas sobre o estado de saúde, condução adeQuada dos problemas de saúde, dentre outros ${ }^{(14)}$.

\section{CONSIDERAÇÕES FINAIS}

A partir da realização do estudo evidenciou-se Que o acesso é um elemento essencial para satisfação das necessidades dos usuários Que procuram os serviços de saúde. A melhoria da Qualidade do atendimento está diretamente ligada às condições de acesso da população às unidades de saúde, sendo identificados como parâmetros importantes de avaliação do acesso o acolhimento, o tempo de espera e a resolubilidade.

A procura pelos serviços de emergência tem ocorrido com certa freqüência, estando associada a concepção de que o hospital é um serviço melhor equipado, Que permanecendo com atendimento 24 horas. Na realidade, a busca pelas unidades hospitalares, onde $22,6 \%$ dos usuários procuram apenas o hospital talvez se deva a deficiências no acesso à atenção básica, levando ao desencadeamento de sucessivas crises hipertensivas.

É notória a necessidade de promover o encontro efetivo das redes assistenciais existentes no município como forma de garantir a continuidade do cuidado e conseqüente integralidade da atenção iniciada na unidade de emergência. O usuário deve ser encarado como sujeito dessa prática, assumindo a co-gestão desse processo.

Observou-se que a cultura da medicalização ainda é muito presente na população. É necessário pensar um modelo de atenção capaz de inverter essa lógica, Que enfoque a saúde não como ausência de doença, mas na perspectiva cidadã e autônoma, Que viabilize a participação de agentes sociais, institucionais e profissionais de saúde, capaz de considerar as diversas práticas de produção de saúde existentes na comunidade para superação de problemas e situações-limite identificadas.

\section{REFERÊNCIAS}

I. Rosa EM, Mezzomo A, Zamboni AP, Pezzi DR. Perfil do diagnóstico e tratamento da crise hipertensiva realizado nos pronto-atendimentos de Caxias do Sul. Rev AMRIGS 2003; 47: 257-61

2. Praxedes J, Santello JL. Emergências e urgências hipertensivas. In: Brandão AA. Hipertensão. São Paulo: Elsevier; 2006. p. 375-90.

3. Varon I, Marik PE. Clinical review: the management of hypertensive crises. Crit Care 2003; 7(5): 374-84.

4. Varon I, Polansky M. Hypertensyve crisis: recognition and management. [cited on 2006 feb 6]. Available: http:// www.uam.es/departamentos/medicina/anesnet/journal/ija/ vol I InI/articles/htncris

5. Travassos C, Viacava F, Almeida CM. Desigualdades geográficas e sociais na utilização de serviços de saúde no Brasil. Cien Saúde Coletiva 2000; 5:133-49.

6. Travassos C, Martins M. Uma revisão sobre os conceitos de acesso e utilização de serviços de saúde. Cad Saúde Pública 2004; 20 (sup 2): 190-8.

7. Souza ACC. Crise Hipertensiva: análise dos casos atendidos na emergência de um hospital municipal de Fortaleza-Ceará, 2006. [dissertação]. Fortaleza: Curso de Mestrado Acadêmico Cuidados
Clínicos em Saúde, Universidade Estadual do Ceará; 2006.

8. Ministério da Saúde (BR). Conselho Nacional de Saúde. Resolução 196: sobre pesquisa envolvendo seres humanos. Brasília: Ministério da Saúde; 1996.

9. Paine S. A report of the finding of a I-year study of waiting times among patients attending an Accident and Emergency department. Accident Emerg Nurs 1994; 2:130-3.

10. Ramos DD, Lima MADS. Acesso e acolhimento aos usuários em unidade de saúde de Porto Alegre. Cad Saúde Pública 2004; 19: 27-34.

11. Paim IS. Modelos de atenção e vigilância da saúde. In: Rouqueroy Z. Epidemiologia e saúde. Rio de Janeiro: Guanabara Koogan; 2003. p.567-86.

12. Valentin IVL, Kruel AJ. A importância da confiança interpessoal para consolidação do programa saúde da família. Cien Saude Coletiva 2007; 12(3): 777-88.

13. Ribeiro RC, Lotufo PA. Hipertensão arterial. Diagnóstico e tratamento da hipertensão. São Paulo: Sarvier; 2005.

14. Núnez-Rocha GM, Guerrero-Flores FI, Salinas-Martinez AM, Villarrela-Rios E, Garza-Elizondo ME. Tiempos de espera? El triage. Una alternativa en el departamento de emergências. Rev Invest Clín 2004; 56: 314-20. 